\title{
CUASI-GRUPOS INTELECTUALES Y PUBLICACIONES COLECTIVAS. EL CASO DE LA REVISTA PAZ E TERRA (1966-1969)
}

RESUMEN: Este artículo analiza al conjunto de intelectuales que participaron en la revista brasileña Paz e Terra. Esta publicación fue un espacio de debate entre cristianos progresistas y marxistas pero tuvo que cerrar prematuramente debido a la persecución que sufrió. Por esta razón solo divulgó diez números entre 1966 y 1969. Nuestra hipótesis es que en ese contexto (Brasil, 1966-1969), las trayectorias de militancia de comunistas y ecumenistas que publicaron en Paz e Terra presentaron paralelismos (como el humanismo, la persecución política, la formación académica) que les permitió encontrarse en un espacio de debate en común y articularse en un cuasi-grupo que, debido al sabotaje de la represión estatal, no alcanzó a conformar un grupo o una red políticoreligiosa. Para contrastarla, en un primer momento indagaremos sobre la teorización en torno a las sociabilidades intelectuales y específicamente, a los cuasi-grupos. Continuaremos con un análisis del contexto histórico brasileño en el que se desarrolló la revista. Posteriormente nos centraremos en la dinámica del ego, Waldo César y el proyecto de la revista Paz e Terra, y culminaremos con las características de los miembros del cuasi-grupo para comparar sus trayectorias biográficas.

Palabras clave: Cuasi-grupo intelectual; revista Paz e Terra; Golpe cívico militar brasileño de 1964; Waldo César.

20 Doctor en Historia por la Universidad Nacional de La Plata; Licenciado en Sociología y pronto a defender su tesis doctoral en ciencias sociales en la Universidad Nacional de Cuyo (UNCuyo). Es Investigador Adjunto del Consejo Nacional de Investigaciones Científicas y Técnicas (CONICET) y docenteinvestigador Categoría II de CONEAU (Argentina), es también director del Centro de Estudios Trasandinos y Latinoamericano de la Facultad de Ciencias Políticas y Sociales de la UNCuyo y director de la Diplomatura en Análisis de Redes Sociales de la Facultad de Educación, UNCuyo. 
60 | Alejandro Paredes

RESUMO: Este artigo analisa ao grupo de intelectuais que participaram da revista brasileira Paz e Terra. Esta publicação foi um espaço de debate entre cristãos progressistas e marxistas, mas teve que fechar prematuramente devido à perseguição que sofreu. Por esta razão, apenas dez números foram relatados entre 1966 e 1969. Nossa hipótese é que neste contexto (Brasil, 1966-1969), as trajetórias dos militantes comunistas e ecumênicos que publicaram em $\mathrm{Paz}$ e Terra apresentou paralelos (como o humanismo, a perseguição, a formação política e acadêmica) que lhes permitiu estar em um espaço comum para debater e se articular em um quase-grupo que, devido a sabotagem da repressão estatal, não conseguiu formar um grupo ou uma rede político-religiosa. Para contrastar a hipótese, ao princípio estudaremos a teorização em torno de sociabilidades intelectuais e, especificamente, aos quase-grupos. Continuaremos com uma análise do contexto histórico brasileiro em que a revista foi desenvolvida. Mais tarde, o artigo vai focar na dinâmica do ego, Waldo Cesar e projecto revista Paz e Terra, e culminar com as características dos membros do quase-grupo para comparar as suas trajetórias biográficas.

Palavras-chave: Grupo quase-intelectual; revista Paz e Terra; golpe cívico militar brasileiro de 1964; Waldo César.

\section{El Cuasi-grupo como sociabilidad intelectual de menor organización.}

$\mathrm{Al}$ analizar el modo en que los intelectuales se han vinculado entre sí, distintos autores han creado, o por lo menos utilizado, múltiples conceptos que en ocasiones describen matices diferentes de estas interacciones. Siguiendo un eje de organización ascendente, que van desde las relaciones entre intelectuales más espontáneas a las más institucionalizadas, podríamos referirnos, entre otros, a los conceptos de "Cuasi-Grupo", "Grupo de Interés", "Grupo Intelectual", "Asociación", "Comunidad de Práctica", "Comunidad Epistémica”, "Redes intelectuales" y, finalmente, "Colegios invisibles".

En este artículo utilizaremos el concepto de cuasi-grupo para abordar el comportamiento de las vinculaciones en torno a la revista Paz e Terra. De todos los conceptos mencionados, el de cuasi-grupo permite estudiar las relaciones entre intelectuales que tienen el menor nivel de organización. Podríamos definirlo como a un conjunto de actores con prácticas e intereses comunes pero cuya configuración resulta casi invisible (MAYER, 1977). Dahrendorf (1957) plantea que los miembros 
Cuasi-grupos intelectuales y publicaciones colectivas. El caso de la revista Paz e Terra (1966-1969) | 61

del cuasi-grupo pueden tener sus intereses en común latentes y, por lo tanto, no ser conscientes que pertenecen a esa estructura social. Por esta razón el cuasi-grupo podría llegar a formar un grupo en el futuro (es decir, que serían un grupo potencial), aunque no necesariamente esto pueda suceder.

Algunas características distintivas separan al cuasi-grupo del grupo de interés, del grupo y de la asociación. El cuasi-grupo está conformado por elementos (sean estos intelectuales, militantes, estudiantes, vecinos, etcétera) que manifiestan intereses similares derivados de una situación común, sin embargo el "grupo de interés" posee, además, una organización, un programa de acción y unos intereses muy definidos como es el caso de los sindicatos, los partidos políticos o los movimientos sociales (GINER, 1970). Un cuasi-grupo también se distingue del grupo o de la asociación porque, en primer lugar, está organizado alrededor de un actor central a través del cual se articula un conjunto de acciones (actionset). Normalmente a este actor central se lo denomina "ego" y puede ser una persona, otro actor similar o un evento. Su importancia es tanta, que al desaparecer este ego, las acciones del cuasi-grupo se diluyen (MAYER, 1977).

En segundo lugar, las acciones de los miembros del cuasi-grupo solo son importantes en cuanto impactan en el ego o en los intermediarios del ego. De hecho, puede no existir interacción entre los miembros del cuasi-grupo de modo que se vinculen solamente con el actor central, dando origen a una estructura estrellada.

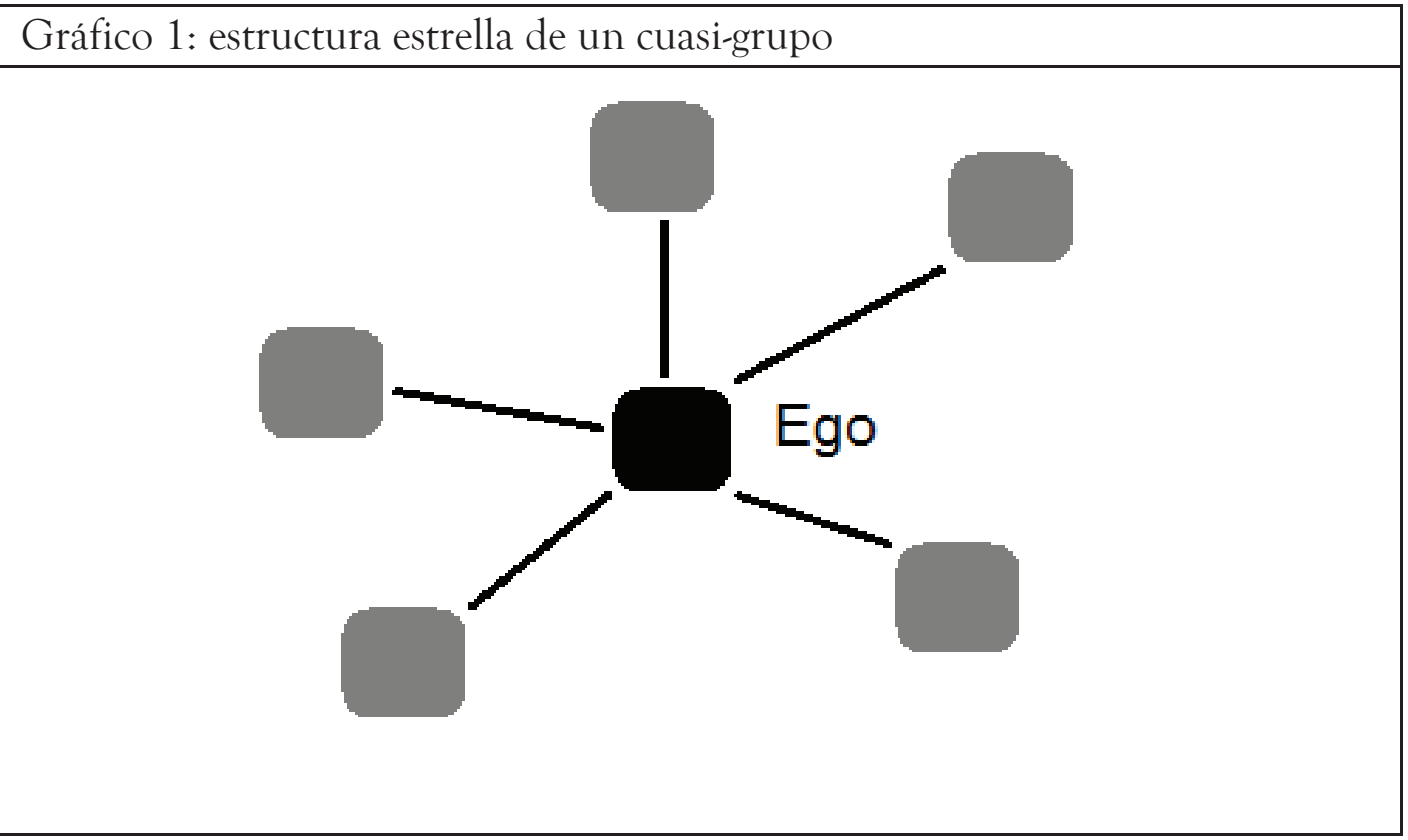


Referencias:

Nodo negro: Ego

Nodos grises: resto de los miembros del cuasi-grupo.

Líneas: interacciones del cuasi-grupo

Fuente: elaboración propia

También pueden existir cuasi-grupos con interacciones entre el resto de los actores, pero cuya importancia es muy baja para la existencia del cuasi-grupo.

Claramente el cuasi-grupo no alcanza los niveles de organización de los otros modos de sociabilidad intelectual más compleja como son la "Comunidad de Práctica", la "Comunidad Epistémica", la "Red intelectual" y el "Colegio invisible".

Por "Comunidades de Práctica" entendemos a un grupo de personas, intelectuales o no, que comparten un interés sobre un tema y que profundizan su conocimiento a través de una interacción continua (WENGER, 2001). En tanto que la "Comunidad Epistémica" es un conjunto de intelectuales que comparten normas y valores, creencias sobre causalidad y nociones de validez, que les cohesionan a la hora de enfrentar un problema (HAAS, 1992). Tanto las comunidades de prácticas como las epistémicas presentan acuerdos consensuados entre todos sus miembros (en relación a sus prácticas o a sus perspectivas teóricas, respectivamente), cierta identidad colectiva, rituales $y$, retomando a Bourdieu, un habitus en común. La red intelectual presenta vínculos de mayor duración, densidad y solidez que permiten la circulación de recursos materiales e inmateriales y límites en los márgenes de acción de sus miembros establecidos a partir de normas codificadas (MOLINA, 2011). Finalmente el Colegio Invisible puede ser entendido como una red intelectual, o al menos una parte de ella, que alcanzado una madurez capaz de sostener procesos formativos que genera a nuevos miembros de la red. Los colegios invisibles, pueden fijar temáticas, los métodos y la terminología en su área del conocimiento (GÓMEZ HERRÁEZ, 2007).

En este artículo analizaremos al cuasi-grupo formado en torno a la revista Paz e Terra, cuyo ego fue Waldo César. Esta revista apareció entre 1966 y 1969, durante la última dictadura cívico-militar brasileña. Su perfil era ecuménico amplio o secular, que analizaba aspectos de la realidad brasileña e internacional (CÉSAR, 2007). Lo novedoso de esta revista es 
Cuasi-grupos intelectuales y publicaciones colectivas. El caso de la revista Paz e Terra (1966-1969) | 63

que fue un espacio convocante para militantes comunistas y cristianos progresistas.

\section{El contexto histórico de la revista}

Tanto el nacimiento y como el declive de Paz e Terra estuvieron íntimamente ligados al contexto político brasileño. El primero de abril de 1964 comenzó en Brasil una dictadura cívico-militar que duró veintiún años. El adjetivo "cívico" hace referencia al apoyo social que obtuvo la dictadura militar diseñada a favor de una élite brasileña y en consonancia con los intereses estadounidenses en la región, en el marco de la Guerra Fría. La victoria de los militares golpistas sería impensable sin la participación de sectores de la sociedad civil liderados por algunos gobernadores, parlamentarios, empresarios, terratenientes, eclesiásticos y los medios masivos de comunicación (PETIT, 2016). De este modo se consiguió la contribución de una militancia de base, como la de católicos conservadores que participaron en la "Marcha da Família com Deus pela Liberdade" o la "Marcha a favor do Ensino Livre" (Codato y Oliveira, 2004). Este apoyo fue también potenciado por la acción de instituciones como el Instituto de Pesquisas e Estudios Sociais (IPÊS). Según Assis (2001), la actividad del IPÊS entre 1962 y 1964 fue decisiva para generar consenso frente al golpe de 1964. Finalmente, también participó una zona gris integrada por aquellos que optaron por un silencio cómplice, una neutralidad pasiva o un retraimiento evasivo para evitar la confrontación y represión del régimen (Rupar, y Rigueiro, 2015; 2017).

Enfrentados a ellos se encontraban los que apoyaron al gobierno depuesto, el amplio espectro de reformistas-populistas, cristianos progresistas (ecuménicos y teólogos de la liberación), los partidos y organizaciones de izquierda y, especialmente, el Partido Comunista Brasileiro. Ellos sufrieron la persecución y los atropellos del régimen. Sólo en el Estado de Pará, alrededor de 300 personas fueron detenidas, entre abril y junio de 1964. La mayoría eran líderes sindicales, militantes del Partido Comunista Brasileño o de Ação Popular (AP) y estudiantes universitarios que fueron liberados en ese mismo año (PETIT, 2016). A partir de 1969 la Operação Bandeirantes centralizó la caza y tortura de los disidentes a través de los Centros de Operações de Defesa Interna (Codis) y los Destacamentos de Operações e Informações: DOIs, que continuaron activos aún después de la Ley de anmistía de 1979 (REIS, 2014; JOFFILY, 2013). 
En este contexto Waldo Cesar organizó la revista Paz e Terra, cuyos autores provinieron de distintas iglesias cristianas, pero también del Partido Comunista Brasileño. Esto pudo suceder debido a que en ese periodo el movimiento ecuménico se identificó con el humanismo crítico, el marxismo y en algunos países, con la Teología de la Liberación (Amat y León, 1996: 30). La revista hace sospechar la existencia de una red intelectual ecuménica-comunista. Esto sería novedoso para Latinoamérica, porque si bien en Asia existieron militantes religiosos, principalmente del Consejo Mundial de Iglesias, que eran miembros de los partidos comunistas de sus países, en América Latina esto no fue habitual ${ }^{21}$.

Para ello indagaremos sobre el origen de la revista, los temas que se debatieron y las trayectorias de militancia de los autores que publicaron más de una vez. Nuestra hipótesis es que en ese contexto (Brasil, 19661969), las trayectorias de militancia de comunistas y ecumenistas que publicaron en Paz e Terra presentaron paralelismos (como el humanismo, la persecución política, la formación académica) que les permitió encontrarse en un espacio de debate en común y articularse en un cuasigrupo que, debido al sabotaje de la represión estatal, no alcanzó a conformar un grupo o una red político-religiosa.

\section{La dinámica del ego: Waldo césar y el proyecto de la revista Paz e Terra}

El fundador de la revista fue Waldo Aranha Lenz César (19222007), quien era parte de un grupo de líderes ecuménicos brasileños que nació al fragor de los movimientos de juventudes protestantes ${ }^{22}$. Se había graduado en 1957 en el Instituto Ecuménico del Consejo Mundial de Iglesias en Ginebra, Suiza (CUNHA, 2007). Posteriormente fue secretario ejecutivo de los departamentos de Mocidade y de Migração e Colonização de la Confederación Evangélica de Brasil. A mediados de los cincuenta el

${ }^{21}$ Eduardo Devés (2016) analiza la acción del Consejo Mundial de Iglesias en la llegada de la Teología de la Liberación a Asia; en tanto que en las trayectorias de asiáticos y africanos se encuentran ejemplos de militantes ecuménicos del Consejo Mundial de Iglesias y que al mismo tiempo, eran miembros de Partidos Comunistas asiáticos (PAREDES, 2013 y 2015)

22 Otros miembros eran: Adauto Araújo Dourado, Benjamin Moraes, Billy Gammon, Boanerges Cunha, Jether Ramalho, Lysâneas Maciel y Rubem Alves (CUNHA, 2007: 140). 
Cuasi-grupos intelectuales y publicaciones colectivas. El caso de la revista Paz e Terra (1966-1969) | 65

movimiento ecuménico priorizó entre sus objetivos la animación de la responsabilidad sociopolítica de los cristianos. En ese contexto, en 1955 el Consejo Mundial de Iglesias financió la creación del Sector de Responsabilidad Social de la Iglesia en el marco de la Confederación Evangélica de Brasil y Waldo César fue su primer secretario ejecutivo (PIMENTEL, 2009). Según José Bittencourt Filho, sus actividades lograron que las iglesias miembro se plantearan un proyecto colectivo para su participación en la realidad nacional sin la influencia de las iglesias estadounidenses (CUNHA, 2007).

Tabla 1: actividades del Sector de Responsabilidad Social de la Iglesia durante la
gestión de W. César
\begin{tabular}{|c|c|l|}
\hline Año & Tipo de actividad & \multicolumn{1}{c|}{ Título } \\
\hline 1955 & Consulta & A responsabilidade social da Igreja \\
\hline 1957 & \multirow{5}{*}{ Conferencias } & $\begin{array}{l}\text { A Igreja e as rápidas transformações sociais no } \\
\text { Brasil }\end{array}$ \\
1960 & $\begin{array}{l}\text { Presença da Igreja na evolução da } \\
\text { nacionalidade }\end{array}$ \\
& Cristo e o processo revolucionário brasileiro \\
\hline 1962 & & \\
\hline
\end{tabular}

Fuente: elaboración propia a partir de CUNHA, 2007: 141

Paralelamente, a partir de 1961, fue secretario adjunto de Iglesia y Sociedad en América Latina (ISAL) que aglutinó a los referentes más importantes del ecumenismo latinoamericano católico y protestante (CONCATTI, 2009: 22-23 y OLIVERA, 2009).

En 1964 fue alejado del secretariado ejecutivo del Sector de Responsabilidad Social de la Iglesia por las repercusiones de la Conferencia del Nordeste de 1962 con el tema Cristo e o Processo Revolucionário Brasileiro (PIMENTEL, 2009). También ese año comenzó a gestarse el Centro Ecumênico de Informação (CEI) cuando en Rio de Janeiro, Waldo César, Francisco de Paula Pereira de Souza, Domício Pereira Mattos y un grupo de alrededor de quince presbiterianos, comenzaron a reunirse regularmente. $\mathrm{Al}$ año siguiente nació oficialmente el $\mathrm{CEI}$ que comenzó a divulgar sus reflexiones a través de folletos, publicaciones en la editorial Tempo e Presença y luego en un suplemento dominical sobre ecumenismo en el Jornal do Brasil. 
En 1966, fue uno de los fundadores y el director de la revista Paze Terra. Waldo César y Luiz Eduardo Wanderley del CEI, contactaron a Ennio Silveira, del Partido Comunista Brasileño, que dirigía la Editorial Civilização Brasileira para la realización de la revista. En ella también participaron algunos miembros de ISAL, como Richard Shaull; Hiber Conteris y Héctor Borrat. En palabras de Waldo César:

... já era depois de 1964. Não podíamos escrever, falar por telefone, tudo era vigiado. Nós viajamos, fomos a São Paulo, fomos a Belo Horizonte, fomos a Volta Redonda, lá falei com padres e pessoas que podiam apoiar para organizar o corpo redatorial da revista que era uma coisa expressiva, com gente desde Alceu Amoroso Lima até esses marxistas que eu falei e outros católicos importantes. Então a revista saiu e teve uma repercussão muito grande. Eram $10 \mathrm{mil}$ exemplares a cada 2 meses, esgotava tudo nas bancas. Ela durou de 1966 a 1969 (EDITORES, 2011, s/p).

La revista Paz e Terra fue sin dudas, el centro de los debates del movimiento ecuménico brasileño y tuvo fuertes lazos con el Consejo Mundial de Iglesias y el Partido Comunista Brasileño. En el primer número, Paz e Terra se presentó como una revista humanista cristiana, que siguiendo la tradición ecuménica, se muestra abierta al diálogo con otras corrientes de pensamiento.

PAZ E TERRA é o campo onde os humanismos, as igrejas e os diálogos dos homens de boa vontade superam as diferenças de estrutura e instituição, raça e credo, cultura e partido, para se encontrarem no reconhecimento da necessidade de defender e promover os valores que se ligam à dignidade e a grandeza da vocação do homem. (PAZ E TERRA N¹, 1966, p.4). 
Cuasi-grupos intelectuales y publicaciones colectivas.

El caso de la revista Paz e Terra (1966-1969) | 67

Cada número de la revista abordó un tema principal y temas secundarios.

Tabla 2: características de cada número de Paz e Terra

\begin{tabular}{|c|c|c|c|}
\hline $\mathbf{N}^{\circ}$ & Año & Temáticas centrales de cada número & $\begin{array}{c}\mathrm{N}^{\circ} \text { de } \\
\text { artículos }\end{array}$ \\
\hline 1 & \multirow{2}{*}{1966} & La Iglesia, ética marxista y cristiana & 24 \\
\hline 2 & & Una visión cristiana y marxista de la historia & 14 \\
\hline 3 & \multirow{3}{*}{1967} & La Juventud, crisis y revolución & 11 \\
\hline 4 & & $\begin{array}{l}\text { América Latina, desarrollo, revolución y } \\
\text { Populorum Progressio. }\end{array}$ & 16 \\
\hline 5 & & La sexualidad & 16 \\
\hline 6 & \multirow{3}{*}{1968} & $\begin{array}{l}\text { Iglesia, el rol del cristianismo en América } \\
\text { Latina }\end{array}$ & 8 \\
\hline 7 & & $\begin{array}{l}\text { Violencia y la vía de la no-violencia para el } \\
\text { cambio social }\end{array}$ & 18 \\
\hline 8 & & $\begin{array}{l}\text { Relación entre tecnología, desarrollo, } \\
\text { mundo, nuclear y ciencia }\end{array}$ & 12 \\
\hline 9 & \multirow{2}{*}{1969} & Educación y universidad & 11 \\
\hline 10 & & El fin de la dictadura de Salazar en Portugal & 10 \\
\hline \multicolumn{3}{|r|}{ (1) } & 140 \\
\hline
\end{tabular}

En los dos últimos números la persecución contra el director de la revista obstaculizó cada vez más su publicación. En 1967 Waldo César había sido detenido e incomunicado por una semana. Posteriormente en una conferencia que dio sobre su viaje a Colombia a una reunión de ISAL y dónde realizó un artículo sobre Camilo Torres publicado en el primer número de Paz e Terra (1966: 243), fue amenazado por un miembro del público. El 14 de diciembre de 1968, al día siguiente del Ato Institucional $n^{\circ} 5$, miembros del ejército fueron a buscarlo a su domicilio, pero él y su esposa se habían escondido en otra casa por consejos de sus familiares. Se inicia así un periodo en que él intenta continuar con sus actividades, pero desde la clandestinidad.

Eu fiquei três meses fora, vinha ao Rio [de Janeiro] escondido, telefonava com outro nome, era uma coisa horrível. Um ano que você se assusta com a própria sombra, porque pode acontecer, e realmente volta e meia apareciam coisas. Era uma perseguição enorme (EDITORES, 2011, $\mathrm{s} / \mathrm{p})$. 
Era muy difícil dirigir la revista así y en el número nueve de Paz e Terra, Waldo César publicó su despedida.

Com este número termino minhas atividades como diretor desta revista. Deixo registrado meu reconhecimento a todos aqueles que confiaram e cooperaram como esta proveitosa experiência cultural e ecumênica - tanto os leitores quanto os membros do conselho de redação - e faço votos de que o debate aberto e honesto entre os homens de boa vontade continue a experiência destes anos (PAZ E TERRA, 1969, p.1).

También Moarcyr Felix, secretario de redacción se solidarizó con Waldo César en su despedida y escribió:

Circunstâncias de sua vida particular tornam impossível a nosso querido amigo Waldo César continuar dando a esta revista a valiosa colaboração que nos prestou ao longo de quase três anos. Lastimando sua ausência, queremos apresentar-lhe de público os protestos sinceros de nosso reconhecimento, certos de que ele, no curso de sua jornada intelectual, será sempre o mesmo batalhador corajoso pela compreensão entre os homens. De nossa parte, garantimos a continuidade dos propósitos que orientaram o surgimento desta publicação, convencidos que estamos da necessidade cada vez mais premente do encontro e diálogo de todas as tendências do humanismo contemporâneo ( $\mathrm{Paz}$ e Terra $\mathrm{N}^{\circ}$ 9, 1969, p.1).

Lamentablemente las condiciones de trabajo se complicaron aún más. Moacyr Félix también se tuvo que esconder varias veces y estuvo preso en más de una ocasión. Su sufrimiento quedó plasmado en la poesía Canção do Exílio Aqui, de 1977 (UHLY, 2006). El número diez fue un mensaje de esperanza. Asfixiada financieramente por la censura, fue la 
Cuasi-grupos intelectuales y publicaciones colectivas. El caso de la revista Paz e Terra (1966-1969) | 69

última edición de Paz e Terra (CUNHA, 2007). El tema central fue el fin de la dictadura de Salazar en Portugal, la más larga hasta el momento del siglo XX. Sus autores eran en su mayoría portugueses aunque, como algunos residían en Brasil, la analogía con la situación brasileña fue constante. Muchos de los artículos eran parte del curso de introducción a la ciencia política promovido por la Pontificia Universidade Católica de São Paulo en septiembre de 1968. La revista analizaba también la retracción del colonialismo portugués.

Waldo César también había participado en la fundación de la editorial Paz e Terra que sí pudo permanecer con sus actividades, pero con un perfil más moderado (MENDONÇA, 2007).

De todos modos, la persecución no pudo evitar que César continuara con su militancia ecuménica. Entre 1970 y 1975 fue miembro del equipo de redacción de la revista Cristianismo y Sociedad (Uruguay) y luego coordinador de la Campaña Mundial contra el hambre de FAO en Santiago de Chile y en Río de Janeiro (institución a la que también estuvo vinculado otro miembro del cuasi-grupo: Luiz Alberto Gómes De Sousa) entre 1979 y 1987 (EDITORES, 2011). Además fue investigador en el ISET-Instituto Superior de Estudos Teológicos y en el ISER-Instituto Superior de Estudos de Religião.

Como escritor puede mencionarse, además de sus numerosos artículos, el romance Tenente Pacífico: a Revolução de 1932, realidade e ficção (2002), la colección de cuentos Tudo tem seu tempo (1985). Los estudios, Para uma sociologia do protestantismo brasileiro (1973) y Pentecostalismo e futuro das igrejas cristãs (1999), escrito junto a Richard Shaull. Fue coordinador e redactor sobre religión en las enciclopedias Delta-Larousse (1970) y Mirador Internacional (1975). Coordinó el libro Protestantismo e imperialismo na América Latina (1969) y tradujo las memorias de Richard Shaull al português: Surpreendido pela graça, en 2003 (MENDONÇA, 2007).

\section{Los miembros del cuasi-grupo}

Paz e Terra nació de la vinculación entre el movimiento ecuménico y el Partido Comunista Brasileño. Los que dirigieron la revista son representantes de ese matrimonio intelectual: El director responsable era Waldo César, un líder del movimiento ecuménico y el secretario era Moacyr Felix, que había sido colaborador en el diario del Partido Comunista Brasileiro y uno de los fundadores del Comando de 
Trabalhadores Intelectuais. Ênio Silveira, miembro del Partido Comunista y propietario de la Editora Civilização Brasileira, que era responsable de la edición de la Revista Civilização Brasileira (RCB), lanzó la revista. Sin embargo, el Partido Comunista Brasileño no tenía influencia directa en la revista ya que publicaron con plena libertad una gran cantidad de cristianos de izquierda que no se sentían parte de la revista anterior (PIMENTEL, 2009, p.6-7).

El consejo de redacción estaba compuesto por Alceu de Amoroso Lima; Frei Pedro Secondi O.P.; Francisco Whitacker Ferreira; Edgar de Godoy Mata Machado; Bernardo Catão; João Dias de Araújo; José Horário Rodrigues; Antônio Otávio Cintra; Jovelino Pereira Ramos; Maria José Brandão Machado; Alfredo Bosi Kühner; Lúcia Ribeiro de Oliveira Ramos; Luiz Alberto Gomes de Souza; Raul Landim Filho; Luiz Eduardo Wanderley; José Paulo Moreira da Fonseca; Glauco Soares de Lima; Padre Francisco Guimarães; Breno Schumann; Jorge César Mota; Esdras Borges Costa y Celso I. de Sylos. Finalmente, los corresponsales extranjeros eran Richard Shaull (Estados Unidos), André Dumas (Francia), Gonzalo Castillo Cárdenas (Colombia), Hiber Conteris y Héctor Borrat de Uruguay.

En la revista se puede observar que 112 autores firmaron 140 artículos. De ellos, 94, es decir el 84\%, publicaron solamente una vez en la revista. Ellos son: A. Bernardo Peres, Afonso Henriques Sacramento, Alceu de Amoroso Lima, Alfredo Marques, Anders Johansson, Ângela Neves, Augusto Aragão, Basil Davidson, Bertrand Russell, Brady Tyson, C.A. Van Peursen, Carl Oglesby, Carlos Drummond De Andrade, Celso Furtado, Cláudio Santoro, Claudius, Conrado Eggers Lan, D. Antônia Ferreira Gomes, Danilo Zolo, Dep Edgar Godoy Mata Machado, Eduardo Alves Da Costa, Ernst Bloch, Fernando Mendes Vianna, Francisco C. Rolim, Francisco Carvalho, Frei Carlos Alberto Cristo, Frei Francisco De Aráujo O.P., Frei Pedro Secondi O.P., Frei Romeu Dale O. P., George Hourdin, Gonzalo Castilho C., H. M. Nussenzveig, Harvey Cox, Hector Borrat, Hugh Hefne, J. Kosinski De Cavalcanti, Jacqueline Skiles, Jayme Abreu, Jean Brun, Jean Lacorix, Joaquim Barradas De Carvalho, John Gerassi, John M. Swomley Jr, Jose Itamar De Freitas, Karl Barth, León Poliakov, Leônidas R. Xausa, Linus Pauling, Luiz Maranhão, Marcel De Clerk, Maria Antônia Fiadeira, Mário Carvalho De Jesus, Mário Moutinho De Pádua, Martin Luther King, Meira Matos, Michael Klare, Michel Dufrenne, Michel Verret, Miguel Urbano Rodrigues, Monsenhor Germán Guzman, Murillo Nunes De Azevedo, Nelson Pilosof, Noronha Filho, Oscar Maggiolo, Raymundo Ozanam De Andrade Sj, Pablo 
Cuasi-grupos intelectuales y publicaciones colectivas. El caso de la revista Paz e Terra (1966-1969) | 71

Piacentini, Padre Camilo Torres, Padre Thomas Melville, Pamela Mills, Paul Lehmann, Paul Louis Landsberg, Paul Tilich, Paulo Freire, Paulo VI, Pietro Nenni, Mario Schenberg, R. Simon S.D.B., Ramón Ramirez Gomez, Raymond Domergue OFM, Régis Debray, Vijaia Laksshmi Pandit, Vitor Ramos, Waldo César, Warwick Esteban, Kerr, Yvan Simonis, Yvon Brés, Leandro Konder, Lauro de Oliveira Lima, Julio de Santa Ana, Otto Maria Carpeaux, Alberto Salvá Contel, Jack Raymond, Donald Duncan y Hugo Weiss.

Solamente 11, casi un 10\%, publicaron dos artículos. Ellos son: André Dumas, Hiber Conteris, J. Leite Lopes, José Honório Rodrigues, Luiz Alberto Gómes De Sousa, Conrado Detrez, Frei Eliseu Lopes O.P., Maria Helena Kühner, Nestor Raul García Canclini, Luiz Eduardo Wanderley y Richard Shaull. Apenas 4, que representan el 3,5\% de los autores, publicaron 3 artículos: Ivan D. Illich, Jovelino Pereira Ramos, Moacyr Felix y Henrique C. De Lima Vaz. Finalmente 3 autores (el 2,5\%), publicaron cuatro artículos. Ninguno era parte del equipo de redacción: Helder Câmara, Pierre Furter, Paul Ricoeur

Estos datos demuestran la muy baja endogamia de la revista, ya que la gran mayoría (el 86,54\%) publicó una sola vez. Tampoco el equipo de redacción publicó mucho. De los 29 miembros del equipo redacción, 16 no publicaron nada, 6 sólo lo hicieron una vez, 6 dos veces y apenas uno publicó tres veces. Waldo César, director de la revista, sólo firmó una publicación, en tanto que Moacyr Felix, quien fue secretario del director y luego director, publicó tres artículos.

Con respecto a la renovación de los autores, el siguiente gráfico analiza la vinculación entre las entregas de Paz e Terra a partir de la publicación de autores en común. 


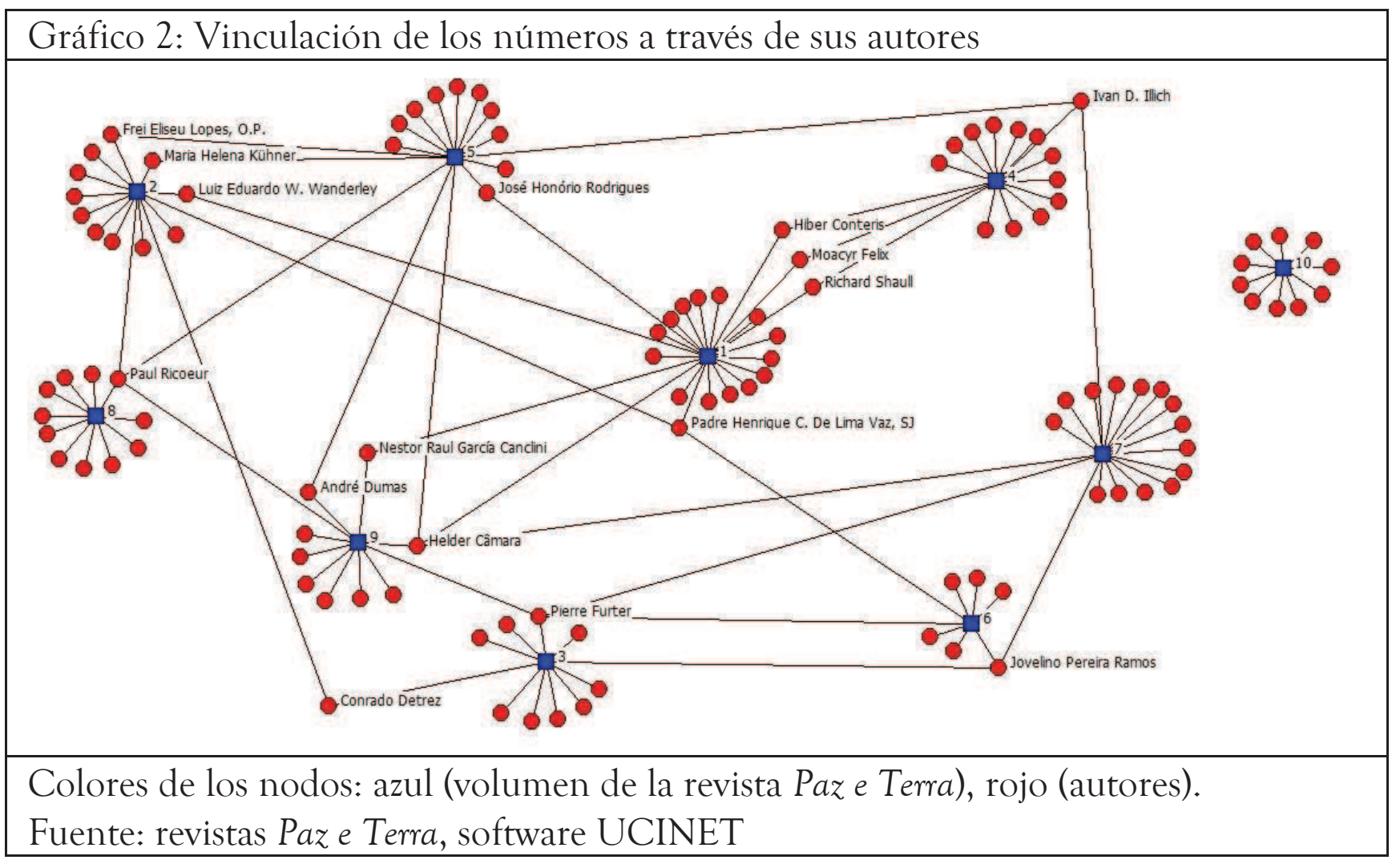

Solamente en el último número de la revista no intervinieron autores que habían escrito en otras ediciones. De todos modos, los autores que han escrito en más de un ejemplar de la revista son pocos (en el gráfico son los nodos que tienen nombre).

Si tomamos a Waldo César y a los 18 autores que publicaron más de una vez, se observan dos trayectorias de militancia distintas, algunas en el campo religioso (ecuménico o eclesial) y otras en el partido comunista (muchos de los intelectuales tienen paralelamente esta militancia). Con respecto a las trayectorias religiosas, ocho tuvieron cargos del movimiento ecuménico, siete eran parte de la jerarquía religiosa de sus iglesias (pastores, sacerdotes u obispos). Sólo seis de ellos no tuvieron cargos en ningún movimiento religioso. En algunos casos, como en el de Moacyr Felix, se debió a su ateísmo y adhesión al partido comunista brasileño. Con respecto a las trayectorias políticas, diez sufrieron algún tipo de persecución, seis tuvieron que exiliarse (en algunos casos exilio interno) y tres estuvieron presos en Brasil, Conteris en Uruguay y Ricoeur en Europa durante la segunda Guerra Mundial. Llama la atención José Honório Rodrigues quien fue director del Instituto Brasileiro de Relações Internacionais entre 1964 y 1968. Finalmente, referido a la adhesión 
Cuasi-grupos intelectuales y publicaciones colectivas. El caso de la revista Paz e Terra (1966-1969) | 73

ideológica al menos en tres casos son autores vinculados al ecumenismo, dos eran teólogos de la liberación y siete eran marxistas.

También pueden encontrarse algunos puntos en común en las trayectorias de los miembros de ambos sectores: todos eran intelectuales muy formados y en su gran mayoría fueron perseguidos por la dictadura cívico-militar brasileña. El cuasi-grupo está conformado por religiosos (Câmara; Lima Vaz; Dumas; Eliseu Lopes; Shaull y Pereira Ramos); escritores (Felix; Conteris; Detrez y Kühner); cientistas sociales (García Canclini; Wanderley; Illich y Waldo César); filósofos (Ricoeur y Furter); un abogado (Gómes De Sousa); un historiador (Honório Rodrigues) y un físico (Leite Lopes). Es un grupo claramente intelectual en el que todos realizaron carreras universitarias y trece de ellos, algún curso de posgrado. Los no-cristianos eran marxistas. Solo hay una mujer, Maria Helena Kühner. En total son once brasileños y ocho extranjeros, de los cuales cuatro vivieron en Brasil (Detrez, Shaull, Illich y Conteris), dos estaban vinculados a la revista francesa cristiana humanista Sprit (Dumas y Ricoeur) y los últimos dos al movimiento ecuménico (Illich y García Canclini). El cuadro siguiente lo detalla: 
74 | Alejandro Paredes

Tabla 3: Comparación de las trayectorias de militancia de los que publicaron más de una vez

\begin{tabular}{|c|c|c|c|c|c|c|c|c|c|c|}
\hline \multirow{3}{*}{ Autor } & \multirow{3}{*}{ País } & \multicolumn{9}{|c|}{ Tópico dominante } \\
\hline & & \multirow{2}{*}{\begin{tabular}{|c|}
$\begin{array}{c}\text { Estudios } \\
\text { de } \\
\text { posgrado }\end{array}$ \\
1
\end{tabular}} & \multicolumn{2}{|c|}{ Religión } & \multicolumn{3}{|c|}{ Política } & \multicolumn{3}{|c|}{ Ideología } \\
\hline & & & 2 & 3 & 4 & 5 & 6 & 7 & 8 & 9 \\
\hline $\begin{array}{l}\text { Néstor García } \\
\text { Canclini }\end{array}$ & Argentina & $X$ & $\mathrm{X}$ & - & - & - & - & $X$ & X & - \\
\hline Ivan Illich & Austria & $\mathrm{X}$ & $\mathrm{X}$ & $X$ & - & - & - & $\mathrm{X}$ & - & - \\
\hline Conrado Detrez & Bélgica & - & - & - & $\mathrm{X}$ & $\mathrm{X}$ & $\mathrm{X}$ & - & $X$ & - \\
\hline Moacyr Felix & \multirow{11}{*}{ Brasil } & $\mathrm{X}$ & 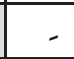 & - & $\mathrm{X}$ & $\mathrm{X}$ & $\mathrm{X}$ & 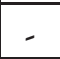 & $\mathrm{X}$ & 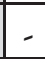 \\
\hline $\begin{array}{l}\text { José Honório } \\
\text { Rodrigues }\end{array}$ & & $\mathrm{X}$ & - & - & - & - & - & - & - & - \\
\hline Waldo César & & $\mathrm{X}$ & $\mathrm{X}$ & - & $\mathrm{X}$ & - & $\mathrm{X}$ & $\mathrm{X}$ & $X$ & - \\
\hline $\begin{array}{l}\text { Luiz Alberto Gómes } \\
\text { De Sousa }\end{array}$ & & $\mathrm{X}$ & $\mathrm{X}$ & - & $\mathrm{X}$ & - & - & $X$ & - & - \\
\hline $\begin{array}{l}\text { Luiz Eduardo } \\
\text { Wanderley }\end{array}$ & & $\mathrm{X}$ & $\mathrm{X}$ & - & - & - & - & $\mathrm{X}$ & $\mathrm{X}$ & - \\
\hline José Leite Lopes & & $X$ & - & - & $\mathrm{X}$ & $\mathrm{X}$ & - & 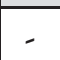 & $\mathrm{X}$ & 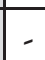 \\
\hline $\begin{array}{l}\text { Jovelino Pereira } \\
\text { Ramos }\end{array}$ & & $\mathrm{X}$ & - & $\mathrm{X}$ & $\mathrm{X}$ & $\mathrm{X}$ & - & $\mathrm{X}$ & - & - \\
\hline $\begin{array}{l}\text { Henrique De Lima } \\
\text { Vaz }\end{array}$ & & $\mathrm{X}$ & - & $\mathrm{X}$ & - & - & - & - & - & - \\
\hline Frei Eliseu Lopes & & - & - & $X$ & - & - & - & $\mathrm{X}$ & - & - \\
\hline Helder Câmara & & 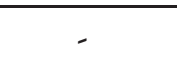 & 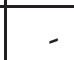 & $X$ & $\mathrm{X}$ & 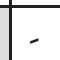 & 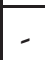 & $\mathrm{X}$ & 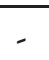 & $\mathrm{X}$ \\
\hline Maria Helena Kühner & & - & - & - & $\mathrm{X}$ & - & - & - & $\mathrm{X}$ & - \\
\hline Richard Shaull & EE.UU. & $\mathrm{X}$ & $\mathrm{X}$ & X & $\mathrm{X}$ & $X$ & - & $\mathrm{X}$ & 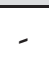 & $\mathrm{X}$ \\
\hline André Dumas & \multirow{2}{*}{ Francia } & - & - & $\mathrm{X}$ & - & - & - & $\mathrm{X}$ & - & - \\
\hline Paul Ricoeur & & - & - & - & - & - & $\mathrm{X}$ & $\mathrm{X}$ & - & - \\
\hline Pierre Furter & Suiza & $\mathrm{X}$ & $\mathrm{X}$ & - & 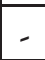 & - & - & $X$ & 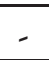 & 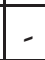 \\
\hline Hiber Conteris & Uruguay & $\mathrm{X}$ & $\mathrm{X}$ & - & $\mathrm{X}$ & $\mathrm{X}$ & $\mathrm{X}$ & $\mathrm{X}$ & 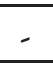 & - \\
\hline
\end{tabular}

Referencias: 1) posgrado, 2) cargos ecuménicos, 3) jerarquía religiosa (pastor, sacerdote, pastor), 4) perseguido, 5) exiliado, 6) preso, 7) ecuménico cristiano, 8) marxista y 9) teólogo liberacionista

Fuente: Elaboración del autor. 
Cuasi-grupos intelectuales y publicaciones colectivas. El caso de la revista Paz e Terra (1966-1969) | 75

\section{Conclusiones}

Viendo la activa militancia de Waldo César puede sostenerse que él era parte del movimiento ecuménico que integró una red activa contra la dictadura cívico-militar integrada por el cristianismo progresista. En ella participaban varios núcleos conformados por los movimientos de juventudes, parte del CEB, el CEI, católicos progresistas y teólogos de la liberación, entre otros. Sin embargo, no puede afirmarse que los autores de artículos publicados en Paz e Terra, llegaron a conformar una red intelectual cristiano-marxista. Este artículo ha podido sostener la hipótesis que, en ese contexto (Brasil, 1966-1969), las trayectorias de militancia de comunistas y ecumenistas que publicaron en Paz e Terra presentaron paralelismos (como el humanismo, la persecución política, la formación académica) que les permitió encontrarse en un espacio de debate en común y articularse en un cuasi-grupo que, debido al sabotaje de la represión estatal, no alcanzó a conformar un grupo o una red políticoreligiosa.

Planteamos que es un cuasi-grupo porque, aunque fueron convocados a publicar por la revista, cristianos y marxistas no llegar a cristalizar una identidad grupal con objetivos consensuados ni una agenda común que la trascendieran. De hecho, una muestra que estamos frente a un cuasi-grupo es la baja endogamia de la revista, el $84 \%$ de los autores publicó solo una vez y el 55\% del equipo de redacción nunca publicó. Esto manifiesta que la temática fue un criterio predominante sobre los potenciales autores y permite sospechar que no nos encontramos ante un núcleo de una red del que Paz e Terra era portavoz sino más bien un espacio convocante a partir del cual se buscaban autores entre las dos corrientes intelectuales (ecumenismo y comunismo) según el tópico de cada entrega.

Es también un cuasi-grupo por la importancia notoria del Ego (Waldo César) en esta formación social. La revista no pudo sostenerse luego del alejamiento de Waldo César, en tanto que tampoco fue posible llegar a una institucionalización de las relaciones establecidas en el marco de la revista, entre el progresismo cristiano y el partido comunista brasileño. Fruto de la eficiente represión del régimen, este intento de vinculación de intelectuales cristianos y comunistas quedó trunco en un cuasi-grupo alrededor de Paz e Terra y de su director, Waldo César. Como 
76 | Alejandro Paredes

afirmó Mendonça (2007: 202) "Não foram tempos de realizações, mas de fracassos. Utopias que se dissolveram no ar".

Finalmente, creemos que estudiar el comportamiento de cuasigrupos intelectuales puede contribuir a los estudios sobre las génesis de redes intelectuales, si las entendemos como sociabilidades partes de protorredes. En este sentido es interesante analizar a cuasi-grupos como el de este artículo, que no evolucionaron hacia formaciones sociales de mayor organización lo que nos permite analizar los aspectos distintivos de las mismas.

\section{BIBLIOGRAFÍA}

ASSIS, Denise. Propaganda e cinema: a serviço do golpe, 1962-1964. Rio de Janeiro: Mauad Editora Ltda. 2001.

CESAR, Waldo. Entrevista: Sociólogo relembra a abertura dos evangélicos para a realidade social brasileira nos anos 60. Ultimato, $\mathrm{N}^{\circ}$ 305, marzo-abril 2007.

CODATO, Adriano y OLIVEIRA, Marcus. A marcha, o terço e o livro: catolicismo conservador e ação política na conjuntura do golpe de 1964. Revista Brasileira de História, 24(47), pp.271-302, 2004.

CONCATTI, Rolando Testimonio cristiano y resistencia en las dictaduras argentinas. El movimiento ecuménico en Mendoza 1963-1983. Buenos Aires: Centro Nueva Tierra, 2009.

CUNHA, Magali do Nascimento. O passado nunca está morto. Um tributo a Waldo César e sua contribuição ao movimento ecumênico brasileiro. Estudos de Religião, XXI (33), pp.136-158, 2007.

CUNHA, Magali do Nascimento. O lugar das produções religiosas de mídia nos processos de oposição e resistência à ditadura civilmilitar no Brasil. compós, 2015. http://www.compos.org.br/biblioteca/compos-2015-dff8e869b6ef-4ae7-b16d-dd0bb16bd446_2891.pdf (consultado, $15 / 3 / 2018)$

DAHRENDORF, R. Las clases sociales y su conflicto en la sociedad industrial, Barcelona: Rialp, 1957.

EDITORES. Waldo César: vida e compromisso com a responsabilidade social da igreja, Novos Diálogos, 20/6/2011, 
Cuasi-grupos intelectuales y publicaciones colectivas. El caso de la revista Paz e Terra (1966-1969) | 77

http://www.novosdialogos.com/artigo.asp?id=596 (consultado, 22/9/2014)

FERREIRA, Jorge y de CASTRO GOMES, Angela. 1964: o golpe que derrubou um presidente pôs fim ao regime democrático e instituiu a ditadura no Brasil. Rio de Janeiro: Civilização Brasileira, 2014.

GINER, Jesús. Conflicto social (Teorías del). Eurotheo, 1970 www. ucm. es/info/eurotheo/diccionario/C/conficto_social_teorias. pdf. (consultado 23/3/2018)

GÓMEZ HERRÁEZ, José María. El pasado cambiante: Historiografía y capitalismo. Siglos XIX y XX, Valencia: Universitat de València, 2007.

HAAS, Peter M. Introduction: Epistemic Communities and International Policy Coordination. International Organization, 46 (1), pp.1-35, 1992.

JOFFILY, Mariana. No centro da engrenagem. Os interrogatórios na Operação Bandeirante e no DOI de São Paulo (1969-1975) Universidade de São Paulo: Tesis Doctoral, 2013.

MENDONÇA, Antonio G. Morre um sonhador-Waldo César (19222007). Estudos de Religião, 21(33), pp.202-204, 2007.

MAYER, Adrian. The Significance of Quasi-Groups in the Study of Complex Societies. In: Leinhardt, Samuel (ed.) Social Networks. A Developing Paradigm. New York: Academic Press, pp.293-318, 1977.

MOLINA, Eugenia. Sociabilidad y redes político-intelectuales: Algunos casos entre 1800 y 1852. Cuadernos del CILHA, 12(1), 19-54, 2011.

OLIVERA, Ademar. Forjando caminos de liberación. La Iglesia Metodista en tiempo de dictadura, Montevideo: Trilce, 2009.

PAREDES, Alejandro "Paralelismos y diferencias en la práctica ecuménica: Un análisis de los vínculos entre América Latina y Asia en la red de publicaciones de Mauricio López (1966-1972)". Asian Journal of Latin American Studies. 26 (2), pp.103-129, 2013.

PAREDES, Alejandro. "Los vínculos de Mauricio López con africanos a través de sus publicaciones colectivas (1966-1972)”. El Acontecer Digital, 7, pp.05-18, 2015.

PETIT, Pere. "El golpe civil-militar, la dictadura y las disputas políticas en el Estado de Pará: 1964-1985”. Revista de Estudios Brasileños, 3(4), pp.24-37, 2016. 
REIS, Daniel A. Ditadura e democracia no Brasil: do golpe de 1964 à Constituição de 1988. Rio de Janeiro: Jorge Zahar, 2014.

RUPAR, B. y RIGUEIRO, J. "50 años del golpe en Brasil: preguntas y debates en torno a la producción historiográfica”. En: Ayala, Mario, Silva, Paulo, Silva, Fabricio y Martins, Fernando (comp.) Anais das IV Jornadas Internacionais de Problemas Latino-Americanos: Lutas, Experiências e Debates na América Latina, Foz do Iguaçu: UNILA, pp.1009-1024, 2015.

RUPAR, Brenda y RIGUEIRO, Julia. Las disputas historiográficas en torno al cincuentenario del Golpe de Estado de 1964 en Brasil: un ejercicio para el abordaje del vínculo entre ciencia histórica y lucha de clases, THEOMAI, 37, pp.61-77, 2017.

UHLY, Steven. Paulo César Fonteles de Lima - Poesia e Ditadura, Revista Eletrônica Literatura e Autoritarismo, 9 (1), 2006 www.ufsm.br/literaturaeautoritarismo/revista/num09/art_01.php (consultado 2/9/2015)

WENGER, Etienne. Comunidades de práctica: aprendizaje, significado e identidad. Barcelona: Paidós. 2001.

Recebido em: 10/07/2018

Aceito em: 24/07/2018 